\title{
3D-Terahertz-Tomography for Material Inspection and Security
}

\author{
Löffler, Torsten \\ SynView $\mathrm{GmbH}$ \\ Glaskopfweg 17, 61479 Glashütten, Germany \\ info@synview.com, www.synview.com
}

\section{Introduction}

The increasing use of novel materials and production technologies increase the demand for new nondestructive testing solutions. At the same time worldwide terrorism leads to a strong request for advanced security technology.

Terahertz and millimeter-wave imaging (THz imaging) is a novel measurement technique, which can serve both application fields. However, in the past $\mathrm{THz}$ imaging was not mature enough to fulfill the numerous industrial and commercial needs. The main limitation was a limited dynamic range leading to a too long image acquisition time.

With the new Synview technology, we overcome the limitations and introduce here a table-top turn-key measurement system which fulfills the needs of scientific and industrial material-testing institutions. Further developments will allow an implementation of the Synview technology in rapid moving production lines and for security screening applications.

Terahertz and millimeter waves are fully harmless electromagnetic waves (similar to radio waves) in the frequency range from $50 \mathrm{GHz}$ to $3000 \mathrm{GHz}(=3 \mathrm{THz})$. The waves can penetrate dielectric or nonconducting materials like plastics, ceramics, paper, cardboard, wood, cloth etc. Metallic or conducting surfaces reflect the waves.

$\mathrm{THz}$ imaging shows the inside of various objects and structures and therefore allows the characterization of hidden or buried interfaces, surfaces or layers. In the security field, suspicious objects hidden underneath the cloth can be made visible.

\section{The Measurement Head}

Contrary to the usually used delicate and costly opto-electronic methods, the Synview technology is based on all-electronic emitters and detectors. This allows us to offer a fast, reliable, and cost-effective solution. Key element of the technology is the SynViewHead 100 and the SynViewHead 300. These units are compact measurement heads which allow the precise measurement of range profiles. Figure 1 shows a photography of the SynViewHead 300.

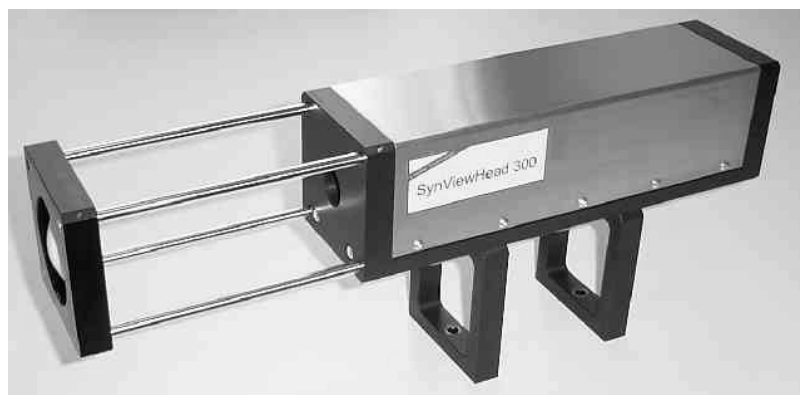

Fig. 1. Photography of the SynViewHead 300.

The operating principle of the SynViewHead is to perform a frequency modulated continuous wave (FMCW) measurement. Via a ramp generator and a VCO (voltage controlled oscillator) a signal with swept frequency is generated at approximately $13-18 \mathrm{GHz}$ with a sweep period of $240 \mu \mathrm{s}$. This signal is used as input for the multiplier chain of the source. A Schottky mixer is employed as heterodyne detector. The system includes a signal generation and data acquisition unit. A specialised data acquisition software allows the calculation of both the absolute range information and the signal phase (with respect to the mean frequency) Appropriate calibration and normalization techniques are included. The unit is available in two different frequency ranges, $60-110 \mathrm{GHz}$ and $230-320 \mathrm{GHz}$. A dynamic range of more than $40 \mathrm{~dB}$ is achieved, for a measurement time as short as $240 \mu \mathrm{s}$. 


\section{The Tomographic 3D Imaging System}

Combining the Synview measurement heads with a fast xy-scanner unit lead to the full 3D imaging system SynViewScan. A photography of the system is shown in Fig. 2. The system allows scanning an area of 600 by $700 \mathrm{~mm}$ in approximately 15 minutes, while the full 3D tomographic information is acquired. A dynamic range of more than $60 \mathrm{~dB}$ is achieved by the system. The SynViewScan 300 System with 230 to $320 \mathrm{GHz}$ operating frequency achieves a spatial resolution of $1 \mathrm{~mm}$, while the SynViewScan 100 with $60-110 \mathrm{GHz}$ operating frequency achieves a spatial resolution of $3 \mathrm{~mm}$. The depth resolution of both systems is on the order of $10 \mu \mathrm{m}$ for a single interface. Individual layers or interfaces can be separated if their spacing is larger than approximately $1 \mathrm{~mm}$.

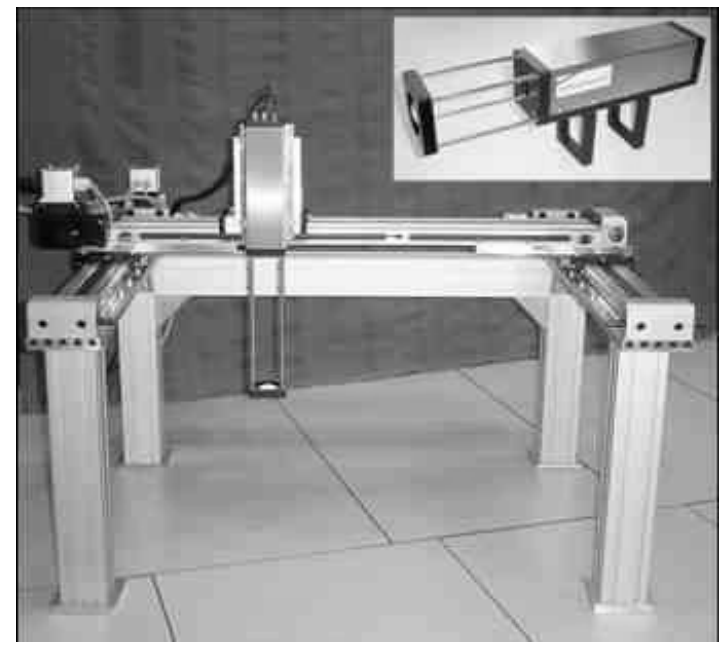

Fig. 2. Photography of the SynViewScan 300.

\section{Application: Package Inspection}

In the following example we demonstrate the capability of the SynviewScan System for the investigation of various objects. The set of the investigated objects is shown in Fig. 3a. It consists of a resolution pattern, a video tape in its cover, a metallic and a ceramic knife, a mobile phone, a DVD in its cover, tablets with/without a cardboard box, razor blades in a cardboard box and a game boy with loudspeaker and batteries inside.
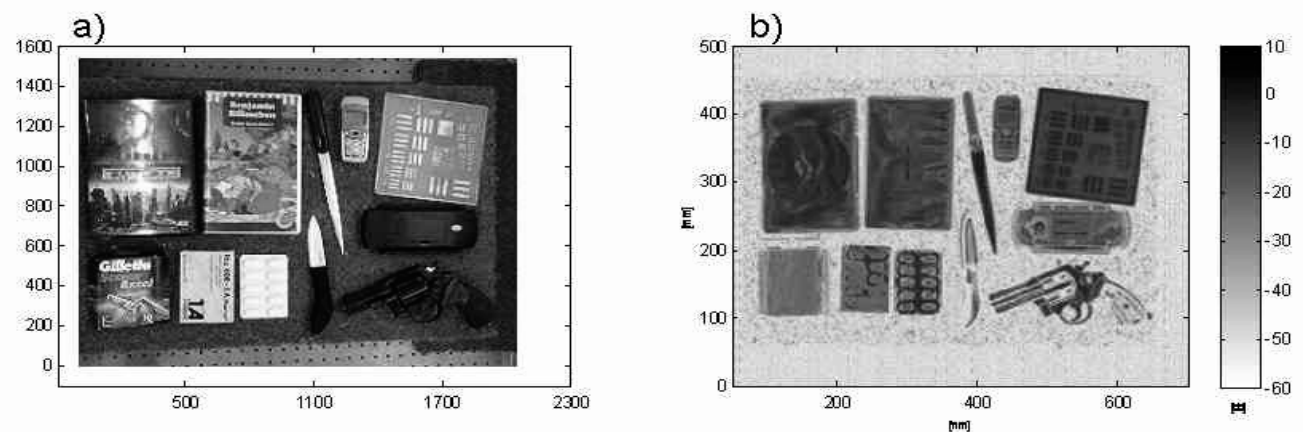

c)

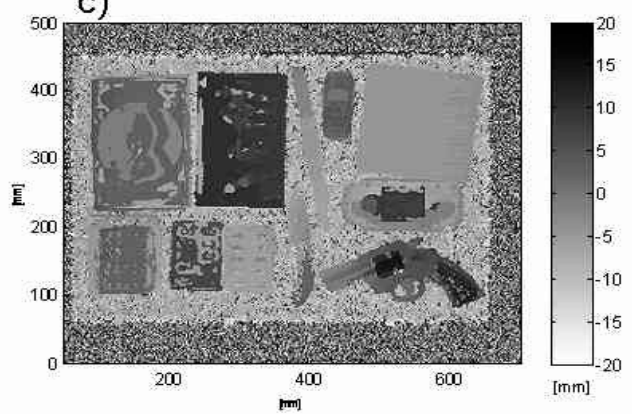

d)

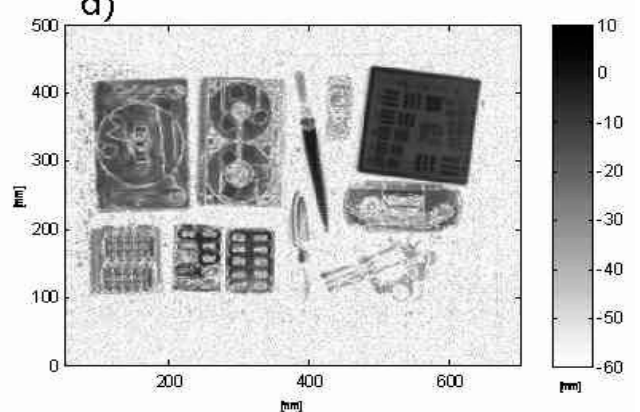

Fig. 3. a) Photography of the arrangement of objects, b)-d) THz data: b) reflectivity, 
c) height, d) tomographic cross section of an inside layer of the objects.

Fig. 3b)-d) show the measurement results of the objects which were placed within the scan area of the SynViewScan 300 system. Three different display modes are shown: the maximum reflectivity of the objects (Fig. 3b), the height of the objects (Fig. 3c) and a tomographic cross section through the inside of the objects (Fig. 3d). The data indicates that although the reflectivity image displays all objects nicely, further imformation can only be achieved by using the Synview 3D measurement capability. For example, the plastic handle of the gun is displayed with a much higher contrast in the height image than in the reflectivity image. The tomographic cross section even allows an inside view of the DVD-box, of the video-tape and of the cardboard boxes with razor blades or tablets. The different appearance of the objects gives a good impression how the system can be used for package inspection, i.e. in the pharmaceutical or food industry. The capability of the technology to explore hidden objects is further demonstrated by placing the whole arrangement of objects inside a large cardboard box as illustrated in Fig. 4b. The results displayed in Fig. $4 \mathrm{~d}$ clearly show that basically the same data quality can be achieved with or without the large cardboard box (Fig. 4c).

a)

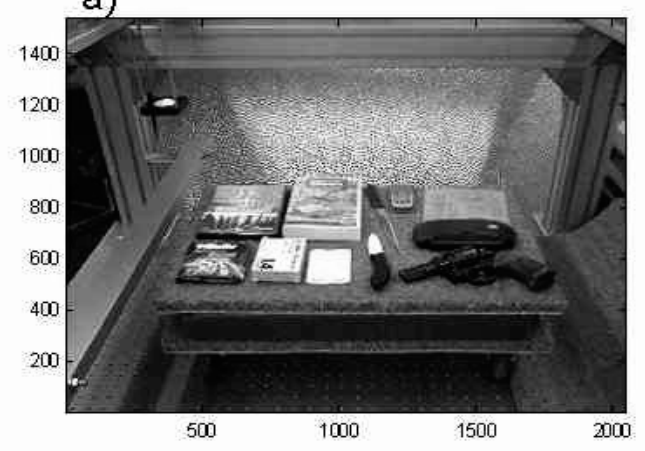

c)

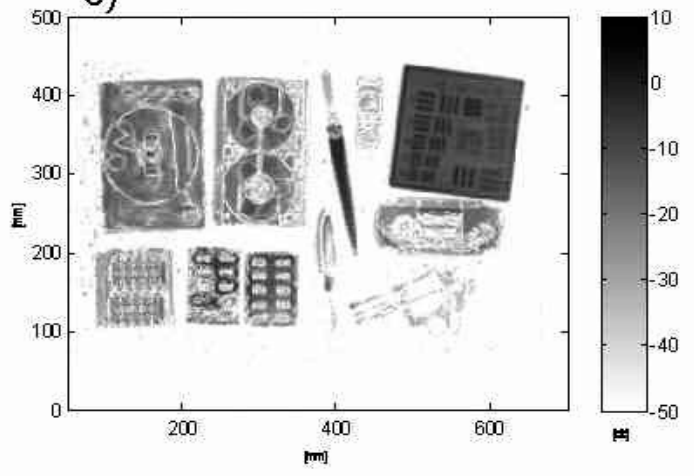

b)
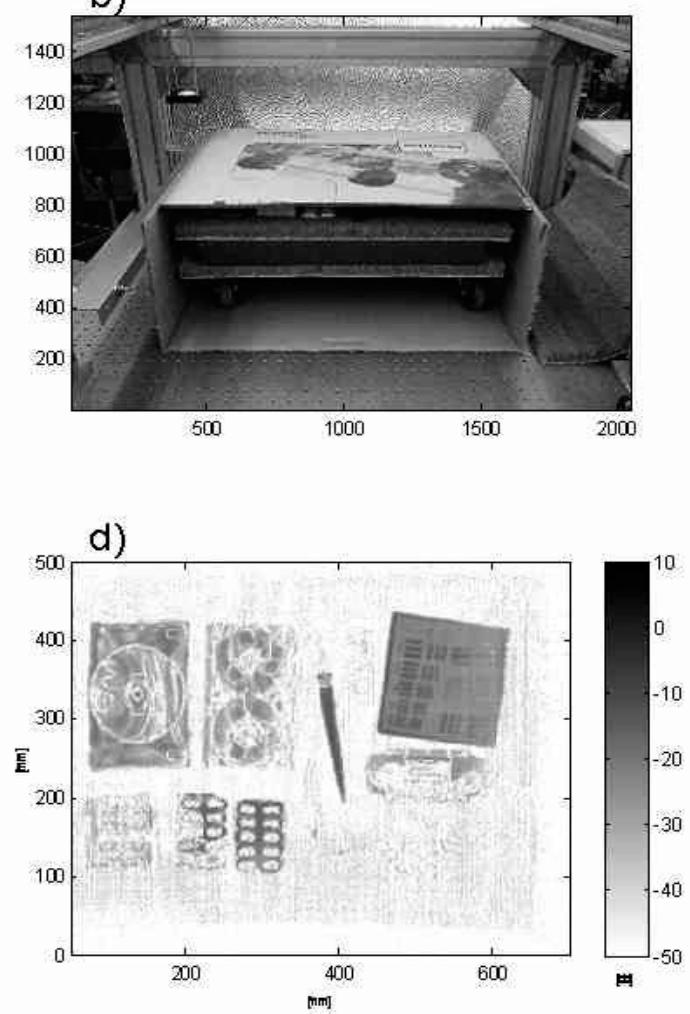

Fig. 4. a) and b) Photography of the measurement scene without (a) and with (b) the large cardboard box. c) and d) THz tomographic cross section(c) without and (d) with the large cardboard box.

\section{Application: Surface Inspection}

The SynViewScan 300 system allows precise measurements of the flatness of rough, hidden or coated surfaces. In order to illustrate this capability, a test sample which consists of a raw machined metal block with an attached "T" out of $\sim 15 \mu \mathrm{m}$ thick aluminum foil was prepared. The height of the surface was determined by employing the phase information of the measured $\mathrm{THz}$ data. As shown in Fig. 5, a change of the surface height in the order of $10 \mu \mathrm{m}$ can easily be measured, even on rough surfaces. 

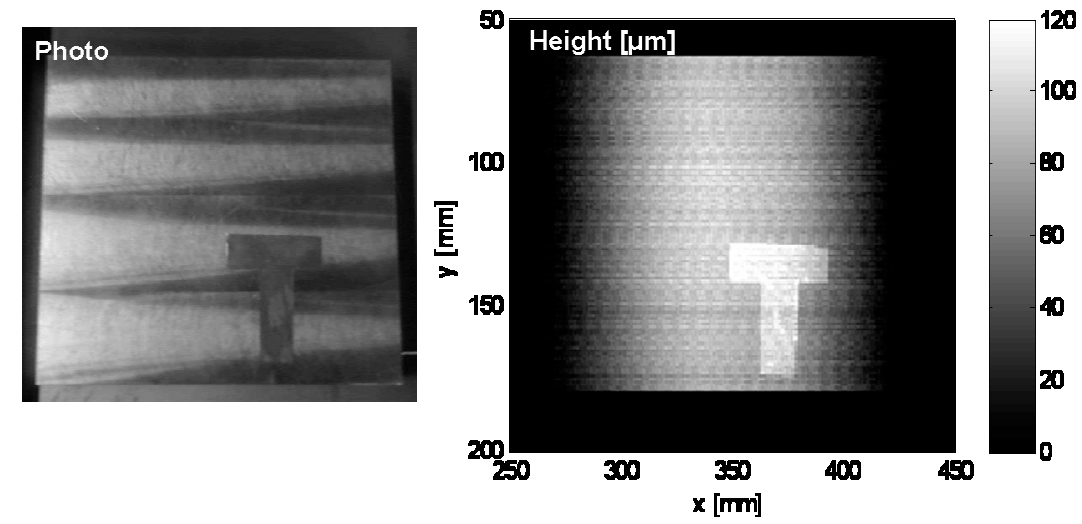

Fig. 5. Photography of the surface height test sample (left) and experimentally determined surface height (right)

\section{Application: Inspection of Sandwich Samples}

Modern elementsin the automotive, aerospace and other industries are composite structures of plastics, foam and several other composite materials. Especially sandwich structures consisting of different materials are very common. In order to demonstrate the possibility to characterise the inside composition of such structures we fabricated a test sample out of various TEFLON and TPX-plastic plates on a SYRODUR foam plate. As glue we used dark wax. Defects in the foam were simulated by drilling two holes in the side of the foam. The photos displayed in Fig. 6 should give a good impression of the fabricated sample.

(a)

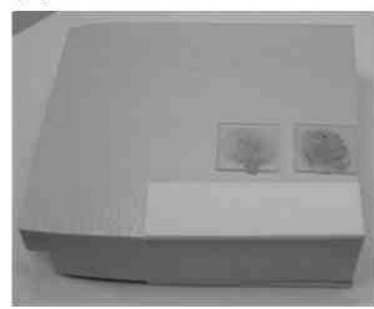

(d)

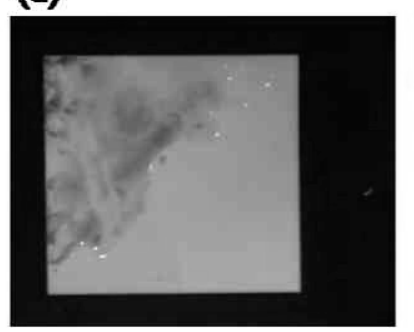

(b)

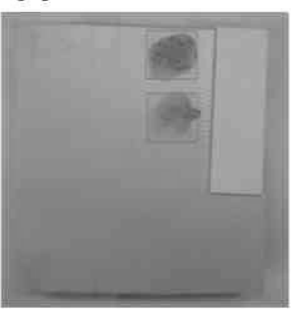

(e) (c)
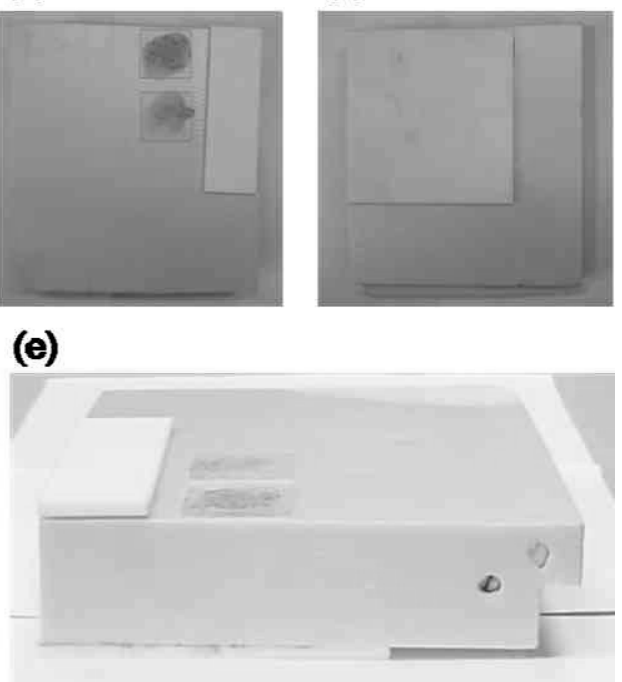

Fig. 6 Photos of the sandwich test sample: (a), (b) top view, (c) bottom view, (d) bottom plate before gluing, and (e) side view.

The sample was mounted upside down so that the side with the three smaller plates was on the bottom side, while the measurement were taken from the top. Fig. 7. gives an overview of the measured data. The upper image shows the maximum reflectivity at each surface point.. The lower image in Fig. 7 shows the cross section of the reflectivity of the sample at the position indicated by the red line in the upper image. 


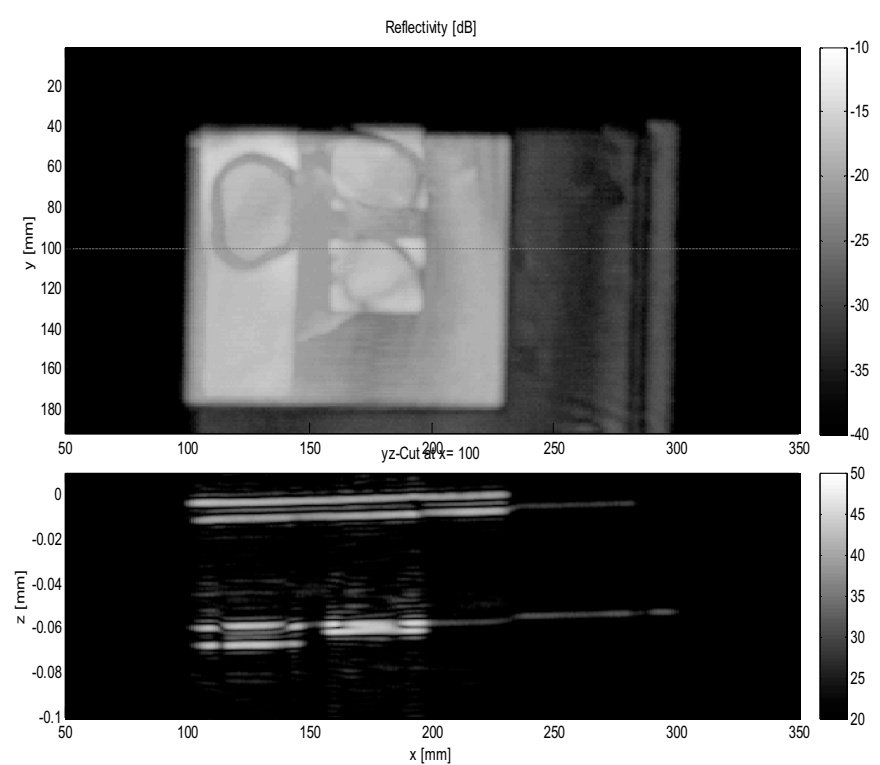

Fig. 7. Top: Maximum reflectivity of the sample, Middle: Cross section of the reflectivity at the position of the red line.

For a detailed analysis of the layered structure the measured volume data was separated in four sections such that each section includes one interface. The boundaries have been chosen manually based on the cross section image (shown in Fig. 8. on the left) and are indicated by dashed lines in the cross section image shown on the right of Fig. 8. For each section the maximum reflectivity (middle column) and the interface height (right column) have been determined. The detailed analysis of the data shows that all interface layers of the sample can be extracted and analyzed separately. Therefore, it is possible to study the homogeneity of the glue and to find air gaps at the interfaces or inside the foam.
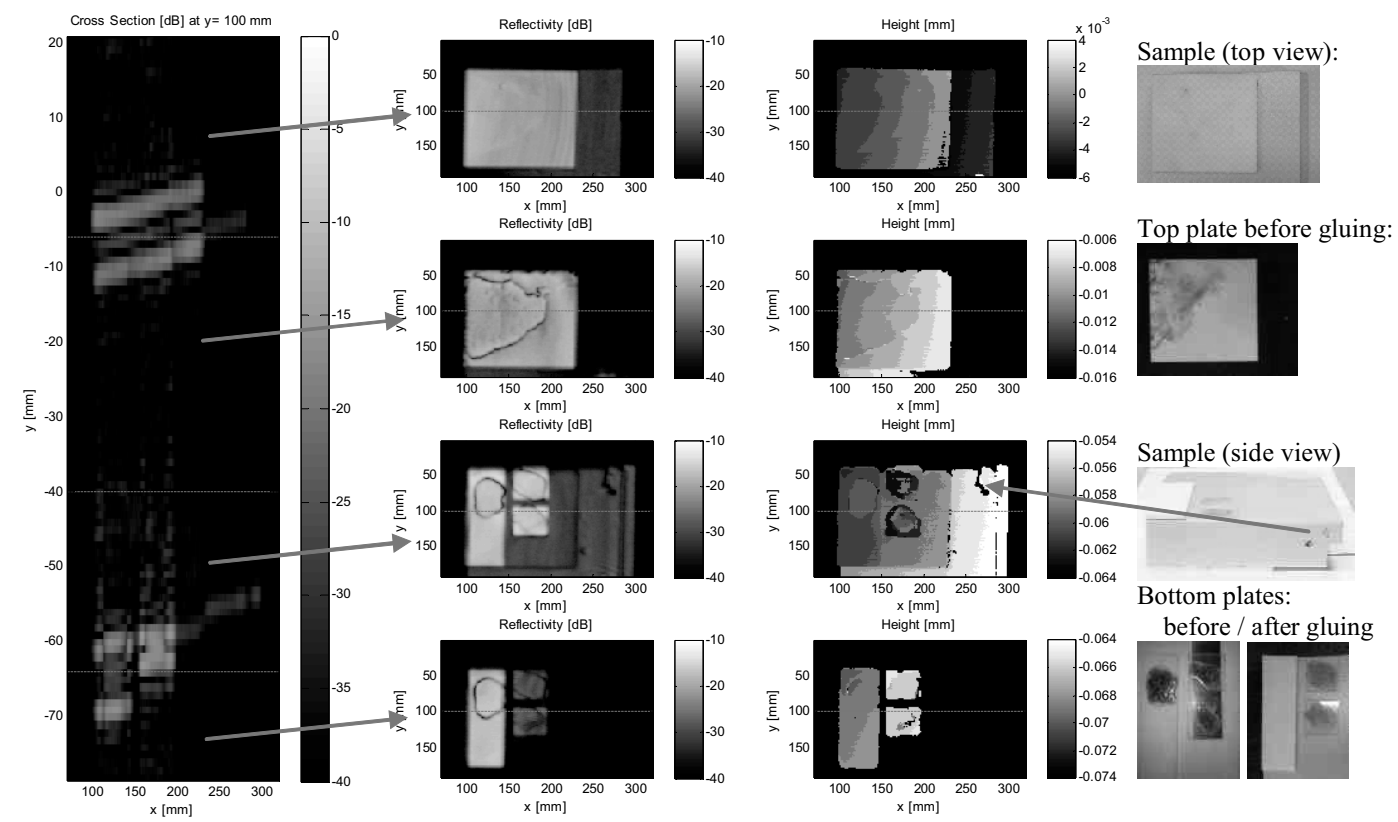

Fig. 8. Left: Cross section of the reflectivity and an indication of the sections. Middle left column: Reflectivity of the individual layer, Middle right column: Height of the individual layer, Right column, photo of the individual layer in the sample. The individual layers from top to the bottom are: a) Top-interface of top plate. b) Glue interface between top plate and foam. c) Glue interface between foam and three bottom plates. d) Bottom interface of three bottom plates. 


\section{Application: Security Exploration}

The SynViewScan 300 system is also ideally suited for the exploration of potential security applications. Although the current measurement time of 10-15 minutes per images is not suitable for real-world implementation, the system still allows to gain very valuable information. One is the possibility to study how various suspicious objects appear in the $\mathrm{THz}$ images under different conditions such as their optical properties, the cloth type or the operating frequency. The achieved 3D data is also a very good base for the development of further image processing techniques to achieve an enhanced visualization, the implementation of object recognition algorithms and the implementation of video overlays or similar techniques to solve the privacy issue. In Fig. 9 the displayed measurement results show the impressive image quality of the Synview technology.
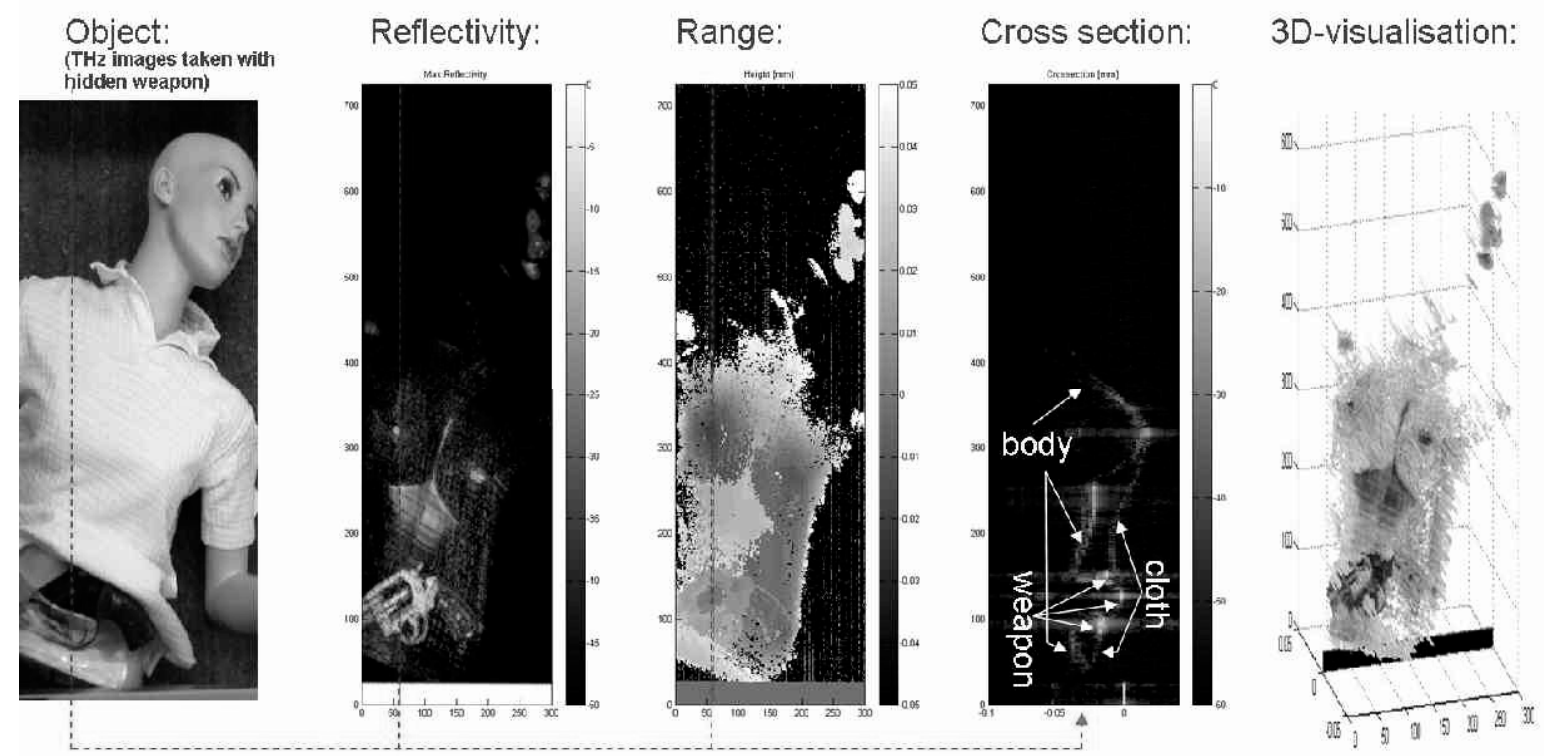

Fig. 9. From left to right: Photo of the mannequin with weapon. (The weapon was hidden under the shirt during the measurements). THz reflectivity image, THz range image, tomographic cross section image at the position indicated by the dashed lines. 3D-visualisation of the THz-data.

\section{Summary and Acknowledgement}

In summary, we have demonstrated a novel all-electronic 3D-tomographic $\mathrm{THz}$ imaging system. The given application examples demonstrate that the system is well suited for non-destructive testing of various $3 \mathrm{D}$ structures, package and surface inspection, and the exploration of security applications. Future developments with multi-element detectors and advanced optic concepts will increase the imaging speed to real-time operation.

We acknowledge financial support from the European Space Agency (ESA-ESTEC) via the project GSTP-4 EE.05 07.1. We are grateful for support from Prof. Viktor Krozer, DTU Copenhagen, Prof. Hartmut G. Roskos from the University Frankfurt and Bernd Hils (MessmO, Königstein). 